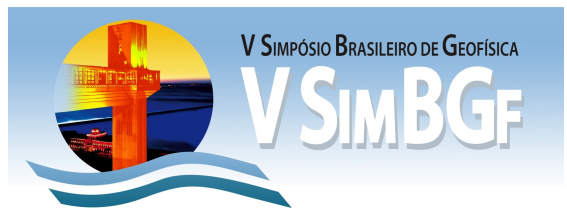

\title{
Espessura crustal usando precursores de pP para telessismos
}

Nataly Aranda*, Marcelo Assumpção

Instituto de Astronomia, Geofísica e Ciências Atmosféricas, Universidade de São Paulo

Copyright 2012, SBGf - Sociedade Brasileira de Geofísica

Este texto foi preparado para a apresentação no V Simpósio Brasileiro de Geofísica Salvador, 27 a 29 de novembro de 2012. Seu conteúdo foi revisado pelo Comite Técnico do V SimBGf, mas não necessariamente representa a opinião da SBGf ou de seus associados. É proibida a reprodução total ou parcial deste material para propósitos comerciais sem prévia autorização da SBGf.

\section{Resumo}

The crustal thickness is a very important property of the crust that is used for different purposes. In this study, we analyze the reflections from the underside of the Moho for an intermediate earthquake occurred in the northern Andes and recorded at teleseismic distances $\left(40^{\circ}-85^{\circ}\right)$ to estimate the crustal thickness at the bounce point. The reflected phase is identified as a precursor of the $\mathrm{pP}$ phase and is called pmP phase. The results obtained show a crustal thickness at the six bounce points of 38.4 , $35.2,35.5,40.1,37.5$ and $42.2 \mathrm{~km}$.

\section{Introdução}

A cordilheira dos Andes é o resultado da subducção da placa de Nazca sob a placa de América do Sul e estende-se perto de $8000 \mathrm{~km}$ desde Venezuela até a Tierra del Fuego. O estudo da espessura crustal nos Andes é importante pois é uma das características pouco conhecidas da crosta na parte norte dos Andes. O conhecimento desta característica é útil para compreender a evolução tectônica desta região, assim como para modelar a propagação de ondas sísmicas, e para melhorar determinação de epicentros regionais.

Um estudo feito por Assumpção et al. (2012) dá uma compilação de espessuras da crosta obtidas por métodos sísmicos para os Andes mostrando que a parte menos estudada é a parte do norte. Fromm et al. (2004) calcularam a espessura crustal sob a região central do Chile e oeste da Argentina. Vários estudos de espessura crustal na parte sul dos Andes foram feitos, como Allmendinger et al. (1990), Regnier et al. (1994) e Gimenez et al. (2009), na parte central dos Andes como McGlashan et al. (2008), e Beck et al. (1996). Na parte norte dos Andes tem-se pouca informação da espessura crustal, a não ser na Venezuela com estudos realizados por Hernandez et al. (2007) e Schmitz et al. (2005).

Devido à ampla atividade sísmica na cordilheira dos Andes especialmente na parte norte dos Andes que é causada pela subducção da placa de Nazca na placa de América do Sul, é possível realizar estudos das ondas sísmicas para determinar a espessura crustal. Neste trabalho é utilizado o precursor de $\mathrm{pP}$ de telessismos para encontrar a espessura da crosta no ponto de reflexão na região dos Andes. Este trabalho é o inicio de um estudo mais amplo que se desenvolverá para encontrar a espessura crustal na parte norte dos Andes entre a Colômbia e o Equador.

\section{Metodologia}

A metodologia aplicada neste estúdio está baseada na identificação de reflexões da parte inferior da crosta (Fig. 1), para sismos com profundidades intermediárias a profundas (>100 km) e com magnitudes Mw>6.0, que são registradas em estações localizadas entre $30^{\circ}$ e $85^{\circ}$ do sismo. Este método foi introduzido por Zhang e Lay (1993) para as reflexões das ondas S e usado por Beck et al.(1996) e McGlasham et al.(2008) na parte central dos Andes.

Neste estudo são analisadas as fases pP e pmP que é a reflexão na Moho. Com a diferença de tempo entre as fases pP e pmP é possível estabelecer a espessura da crosta com a seguinte relação:

$$
t_{p P}-t_{p m P} \approx 2 \mathrm{~h} \sqrt{V_{p c}^{-2}-p^{2}}
$$

onde $\mathrm{h}$ é a espessura da crosta, $\mathrm{V}_{\mathrm{pc}}$ é a velocidade média da onda $P$ na crosta e $p$ é o parâmetro de raio das fases pP e pmP. Este parâmetro foi assumido o mesmo para as duas fases como foi feito por Zhang e Lay (1993), Zandt et al. (1994) e McGlashan et al. (2008).

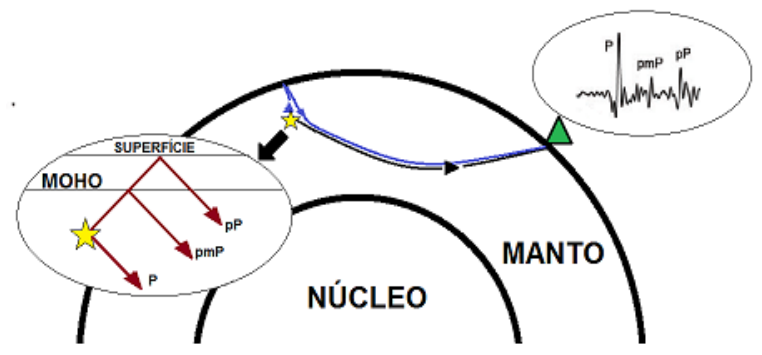

Figura 1- Ilustração das reflexões das fases das ondas $P$, modelo tomado de McGlashan et al. 2008.

Para calcular a diferença de tempo entre as fases pP e pmP foi utilizado o programa SAC(seismic analysis code). Primeiro foi determinado o tempo de chegada da onda $\mathrm{pP}$ de um mesmo evento registrado em estações diferentes. Esse tempo de chegada foi alinhado para todas as estações e foram somados todos os sismogramas para realçar a chegada da onda pmP, que é observada como precursora da onda pP (Fig. 2).

Do mesmo modo é necessário determinar o ponto de reflexão que é o ponto para o qual é encontrada a espessura da crosta. Isso foi feito com o programa TauP o qual mostra o parâmetro $p$ para distâncias focais e profundidades dadas. Com o parâmetro $p$ foi determinada 
a distância do ponto de reflexão e com o azimute do sismo foram determinadas as coordenadas desse ponto.

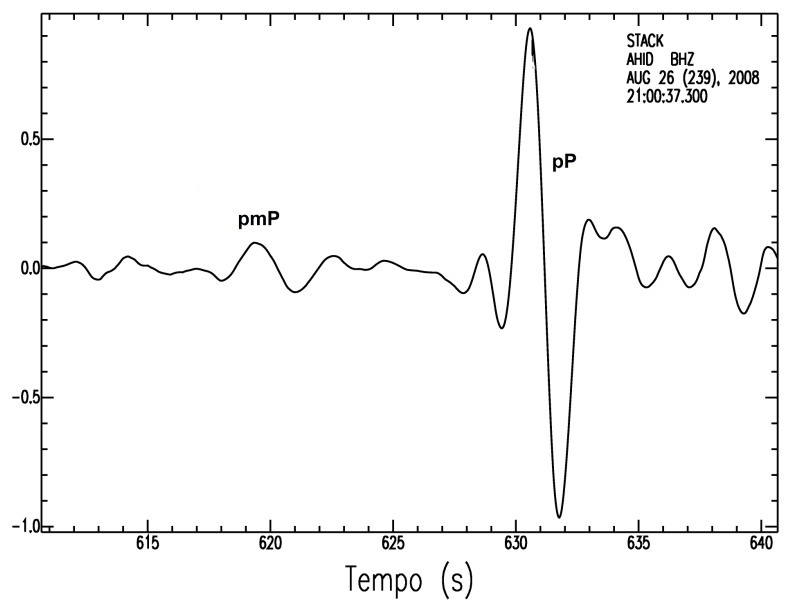

Figura 2- Fases pmP e $p P$ da soma dos sismogramas das 10 estações analisadas para o sismo ocorrido o dia 26 de agosto e 2008.

\section{Resultados}

Os sismos estudados ocorreram os dias 24 de agosto de 2011 em $\left(74.51^{\circ} \mathrm{W}, 7.64^{\circ} \mathrm{S}\right)$ com magnitude de $6.8 \mathrm{e}$ profundidade de $147 \mathrm{~km}$, o dia 26 de agosto de $2008 \mathrm{em}$ $\left(74.5^{\circ} \mathrm{W}, 7.5^{\circ} \mathrm{S}\right) \mathrm{com}$ magnitude de 6.3 e profundidade de $152 \mathrm{~km}$ e o dia 2 de agosto de 2012 em ( $74.24^{\circ} \mathrm{W}$, $8.37^{\circ} \mathrm{S}$ ) com magnitude de 6.1 e profundidade de 143.3 $\mathrm{km}$. Para o sismo do dia 26/08/2008 foram analisados 10 sismogramas de diferentes estações, localizadas a uma distancia epicentral entre $40^{\circ}$ e $65^{\circ}$ do evento. Na figura 3 são mostradas as estações e o local do epicentro deste sismo.

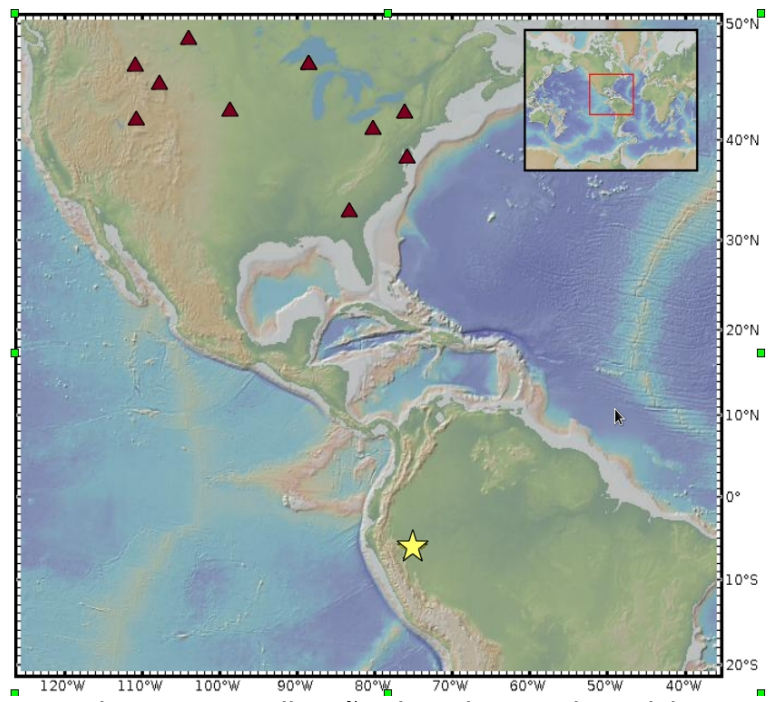

Figura 3- Localização do epicentro (estrela) e das estações analisadas (triângulos) para o sismo ocorrido o dia 26 de agosto de 2008.

Para cada estação foi encontrada a chegada da onda pP com o programa SAC (Seismic Analysis Code), os resultados obtidos para o sismo do 26 de agosto de 2008 são mostrados na figura 4 onde é possível observar os sismogramas para as 10 estações analisadas, alinhados no pico maior da onda pP. Foi feita a soma dos sismogramas para encontrar o precursor da onda $\mathrm{pP}$, esse precursor é tomado como a onda pmP, o resultado desta soma é exibido na Figura. 2 onde é possível observar entre $618 \mathrm{~s}$ - 621s um pequeno pico que é a chegada da onda pmP.

$\mathrm{Na}$ figura 4 pode-se ver que nem todos os sismogramas têm um pico definido na posição da chegada da onda pmP, por esta razão é importante fazer o empilhamento dos sismogramas para assim poder identificar melhor a fase $\mathrm{pmP}$.

O tempo obtido entre as ondas pmP e pP foi de $11.19 \mathrm{~s}$ para o sismo do 26/08/2008. A velocidade média da crosta foi tomada como $\mathrm{V}_{\mathrm{pc}}=6.45 \mathrm{~km} / \mathrm{s}$, reportada como a velocidade média da crosta para regiões estáveis (Christensen \& Mooney 1995). O parâmetro de raio foi calculado como a média dos parâmetros de raio dos 10 sismogramas: $p=7.482 \mathrm{~s} /{ }^{\circ}$. Com estes valores na equação 1 , foi calculada a espessura da crosta em 40.1 $\mathrm{km}$.

O ponto de reflexão do sismo do dia 26/08/2008, que é o lugar onde foi calculada a espessura, está localizado em $\left(74.75^{\circ} \mathrm{W}, 6.70^{\circ} \mathrm{S}\right)$ muito perto do epicentro (foi calculado usando o parâmetro $p$ e o azimute médio das 10 estações). Os resultados obtidos para os três sismos analisados são mostrados na Tabela 1.

Tabela 1: Resultados das espessuras obtidas em seis pontos de reflexão para os três sismos analisados.

\begin{tabular}{|c|c|c|c|c|}
\hline Evento & $\begin{array}{r}\text { Espessura } \\
\text { crustal }(\mathrm{km})\end{array}$ & Ponto de reflexão & $\begin{array}{r}\text { Azimute } \\
\text { médio }\end{array}$ & \begin{tabular}{|r|} 
Número de \\
estações
\end{tabular} \\
\hline \multirow{3}{*}{$\begin{array}{l}08 / 26 / 08 \\
(74.47 \mathrm{~W}, 7.54 \mathrm{~S}) \\
\mathrm{H}=152 \mathrm{~km}, \mathrm{Mw}=6.2\end{array}$} & 40.1 & $(74.75 \mathrm{~W}, 6.70 \mathrm{~S})$ & 342 & 10 \\
\hline & ruidoso & $(74.39 \mathrm{~W}, 7.46 \mathrm{~S})$ & 68 & 4 \\
\hline & & & & \\
\hline \multirow{3}{*}{$\begin{array}{l}08 / 24 / 11 \\
(74.51 \mathrm{~W}, 7.64 \mathrm{~S}) \\
\mathrm{H}=147 \mathrm{~km}, \mathrm{Mw}=6.8\end{array}$} & 38.4 & $(74.61 \mathrm{~W}, 6.97 \mathrm{~S})$ & 352 & 7 \\
\hline & 35.2 & $(73.75 \mathrm{~W}, 7.47 \mathrm{~S})$ & 78 & 4 \\
\hline & 35.5 & $(74.21 W, 7.29 \mathrm{~S})$ & 42 & 4 \\
\hline \multirow{3}{*}{$\begin{array}{l}08 / 02 / 12 \\
(74.24 \mathrm{~W}, 8.37 \mathrm{~S}) \\
\mathrm{H}=143 \mathrm{~km}, \mathrm{Mw}=6.1\end{array}$} & $37.5 ?$ & $(74.46 \mathrm{~W}, 7.72 \mathrm{~S})$ & 341 & 14 \\
\hline & 42.4 & $(73.87 W, 8.06 \mathrm{~S})$ & 50 & 10 \\
\hline & ruidoso & $(74.16 \mathrm{~W}, 7.65 \mathrm{~S})$ & 6 & 5 \\
\hline
\end{tabular}

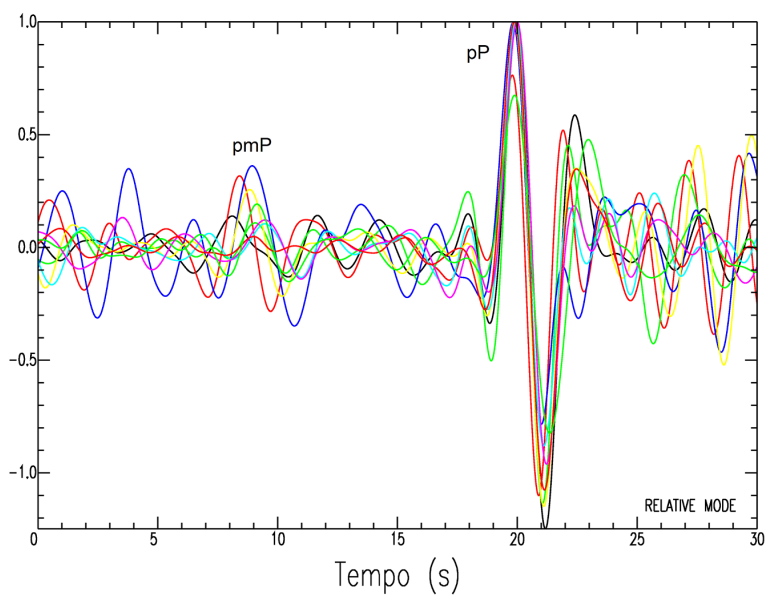

Figura 4- Fases pmP e pP dos 10 sismogramas analisados do sismo ocorrido o 26 de agosto de 2008 . 


\section{Discussão e Conclusões}

O método aplicado neste estudo foi usado anteriormente no estudo da espessura da crosta por Zhang e Lay (1993), Zandt et al. (1994) e McGlashan et al. (2008), mas observou-se que nem todos os sismogramas que foram analisados mostraram o precursor da onda $\mathrm{pP}$ (onda pmP) bem definido. Por isto é importante fazer a soma dos sismogramas antes de encontrar o tempo entre as ondas analisadas. Neste trabalho vemos que para os sismogramas analisados nem todos mostram a fase pmP bem definida (Fig. 4), mas ao fazer a soma (Fig 2) vemos bem definido o ponto de chegada da onda pmP. Essa característica dos sismogramas pode ser devida a variabilidade da refletividade na Moho, ou seja tem algumas zonas com baixa refletividade na Moho ou presença de ruído nos sismogramas.

Em síntese, a espessura da crosta obtidas que são mostradas na Tabela 1 . são resultados que estão de acordo com estudos anteriores feitos para esse lugar. A velocidade média da crosta utilizada para converter a diferença pP-pmP em espessura foi de $6.45 \mathrm{~km} / \mathrm{s}$ como reportada por Christensen \& Mooney (1995).

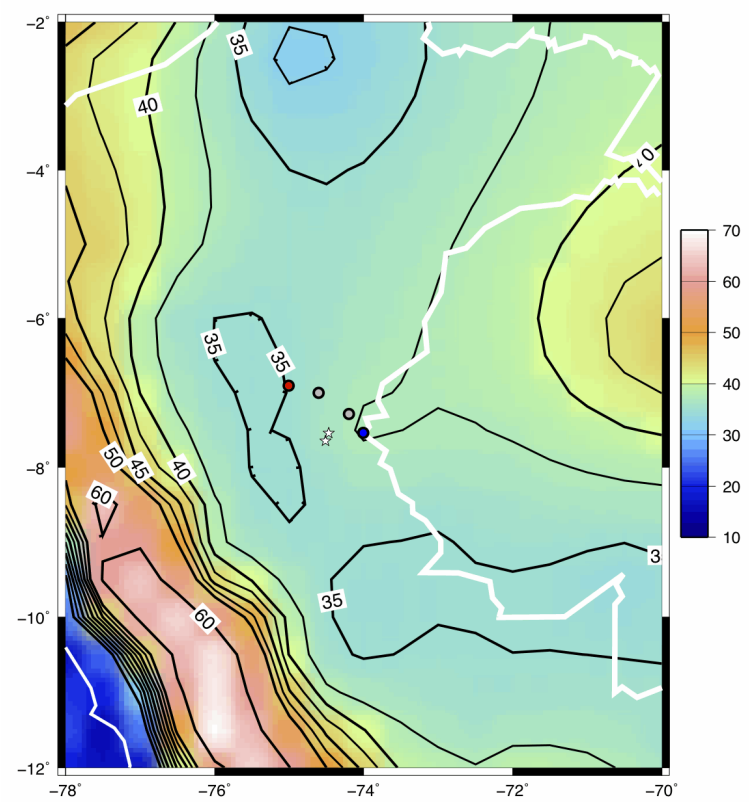

Figura 5- Resíduos das espessuras obtidas com pmP. pP (círculos) de dois sismos (estrelas). Os contornos referem-se à espessura crustal de Assumpção et al. (2012) interpolados de outros dados sísmicos. Os círculos cinza representam as diferenças entre as espessuras pmP-pP e as esperadas (contornos), i.e., resíduos menores que $2.5 \mathrm{~km}$; o círculo vermelho significa resíduo entre 2.5 e $5 \mathrm{~km}$; e o círculo azul entre -2.5 e $-5 \mathrm{~km}$.

Devido à pouca informação encontrada da espessura da crosta da parte norte dos Andes, é importante continuar um estudo detalhado para esta zona. Outros sismos estão sendo analisados e alguns resultados preliminares estão mostrados na Figura 5 Estes resultados estão de acordo com os valores esperados pela compilação de Assumpção et al. (2012) e são o inicio de uma ampla investigação da espessura crustal da parte norte dos Andes localizada na Colômbia e no Equador.

\section{Referências}

Allmendinger, R. W., Figueroa, D., Snyder, D., Beer, J., Mpodozis, C., \& Isacks, B.L., 1990. Foreland shortening and crustal balancing in the Andes at $30{ }^{\circ} \mathrm{S}$ latitude. Tectonics, 9, 789-809.

Assumpção, M., Feng, M., Tassara, A., Jordia , J., 2012, Developing models of crustal thickness for South America from receiver functions and surface wave dispersion tomography. Tectonophysics, submitted.

Beck, S. L., Zandt, G., Myers, S. C.,Wallance, T. C., Sielver, P. G., \& Drake, L., 1996. Crustal-thickness variations in the central Andes. Geology, 24, 5, 407-410.

Christensen, N. \& Mooney, W. D., 1995. Seismic velocity structure and composition of the continental crust: A global view. J. Geophys. Res., 100, B7, 9761-9788.

Fromm, R., Zandt, G. \& Beck, S. L., 2004. Crustal thickness beneath the Andes and Sierras Pampeanas at $30^{\circ} \mathrm{S}$ inferred from $\mathrm{Pn}$ apparent phase velocities. Geophys. Res. Lett., 31, L06625.

Gimenez, M. E., Braitenberg, C., Martinez, M. P., \& Introcaso, A., 2009. A comparative analysis of seismological and gravimetric crustal thicknesses below the andean region with Flat subduction of the Nazca plate. International Journal of Geophysics, 2009, article ID 607458.

Hernandez, P. O., Von Frese, R., \& Jeong Woo, K., 2007. Crustal thickness variations and seismicity of northwestern South America. Earth Sci. Res. J., 11, 1, 8194.

McGlashan, N.,Brown, L., \& Kay, S., 2008. Crustal thickness in the central Andes from teleseismically recorded depth phase precursors. Geophys. J. Int., 175, 1013-1022.

Regnier, M., Chiu,J. M., Smalley, R., Isacks, B. L., \& Araujo M., 1994. Crustal thickness variation in the Andean foreland, Argentina, from converted waves. Bull. Seismol. Soc. Am., 84, 1097- 1111.

Schmitz, M., (13 co-autores), 2005. Crustal thickness variations in northern Venezuela from deep sismic observations. $6^{\text {th }}$ International symposium on Andean geodynamics. 655-658.

Zandt, G., Velasco, A. \& Beck, S. 1994. Composition and thickness of the southern altiplano crust, Bolivia, Geology, 22, 1003-1006.

Zhang, Z. \& Lay, T., 1993. Investigation of upper mantle discontinuities near northwestern Pacific subduction zones using precursors to sSH, J. Geophys. Res., 98, 4389-4406. 